\title{
The investigation of the spectra of solar events observed in October-November 2003.
}

\author{
Irene V. Arkhangelskyaja ${ }^{1}$, A.I. Arkhangelsky ${ }^{1}$, Yu. D. Kotov ${ }^{1}$, \\ and S. N. Kuznetsov ${ }^{2}$ \\ ${ }^{1}$ Astrophysics Institute, Moscow State Engineering Physics Institute (State University) \\ Kashirskoe shosse 31, Moscow, 115409 Russia email: irene.belousova@usa.net \\ ${ }^{2}$ Scobeltsyn Institute of Nuclear Physics, Moscow State University, 119899 Russia
}

\begin{abstract}
More than 30 active regions were observed on the Sun during October-November 2003 by SOHO. Approximately half of such regions induced hard X-ray and $\gamma$-ray emission and some solar flares with class $\mathrm{M}$ and $\mathrm{X}$ were detected during this time by GOES, RHESSI and other experiments. 5 solar flares were detected by AVS-F instrument onboard CORONAS-F satellite. Nuclear $\gamma$-emission lines were detected during some flares observed in this period, in particular, during all flares on October 292003.
\end{abstract}

More than 30 active regions were observed on the Sun during October-November 2003 by SOHO. Four regions $(0484,0486,0488,0490)$ induced hard X-ray and $\gamma$-ray emission and 14 solar flares with class $\mathrm{M}$ and $\mathrm{X}$ were detected during this time by GOES, RHESSI and other experiments. 5 solar flares were detected by AVS-F instrument (apparatus description see Arkhangelsky (1999)) onboard CORONAS-F satellite. Two of them (23.10.2003 at 08:08:00 UT, 26.10.2003 at 05:57:00 UT ) were registered in the polar cap. Flare 04.11.2003 at 19:29:00 were observed both in equatorial region and in polar cap. For one flare (26.10.2003 at 23:37:00 UT) the AVS-F device observed only the end of the flare without significant presence of nuclear $\gamma$-lines. Now we conduct more carefully analysis of the spectra of these four flares.

The intensive flare was detected by devices onboard GOES-satellite series (class X10, ball 2B, NOAA region 0486, S15W02, 29.10.2003 from 20:37 to 21:01 UT) ( sec.noaa.gov) with maximum emission in nuclear $\gamma$-lines region. This flare was observed by AVS-F instrument in the equatorial orbit region in 20:39:00-20:58:00 UT. The CORONAS-F satellite came out from radiation belt at 20:39 UT and came in again at 20:58 UT. There are not significant changes in charged particles (electrons in the energy ranges of 0.3-0.6, 0.6-1.5 MeV and 1-5 MeV protons on MKL ( Kuznetsov et al. (1995)) data) fluxes affected on the $\gamma$-ray temporal profile during this time interval. The spectrum for 20:39:07-20:55:00 UT time interval based on AVS-F data is shown at Figure 1.a. There are 5 nuclear $\gamma$-line complexes in this spectrum: 0.81-0.94, 1.51-1.74, 2.9-3.4, 4.0-5.0, 5.3-6.9 $\mathrm{MeV}$ corresponding ${ }^{56} \mathrm{Fe},{ }^{24} \mathrm{Mg}+{ }^{20} \mathrm{Ne}+{ }^{28} \mathrm{Si},{ }^{20} \mathrm{Ne}+{ }^{16} \mathrm{O},{ }^{12} \mathrm{C}$ and ${ }^{16} \mathrm{O}$ lines accordingly and complex 2.14-2.64 MeV corresponding to neutron capture line. These lines were seen during whole flare. There are some weak lines in the region of $0.3-0.6 \mathrm{MeV}\left(\mathrm{e}^{+} \mathrm{e}^{-}\right.$ for example) probably. These $\gamma$-lines complexes were observed in the spectra of number solar flares, for example 27.10.1991 ( Mandzhavidze \& Ramaty (1992)). Figure 1.b shows temporal profiles of 29.10.2003 flare in energy ranges corresponding to mentioned above lines and in continuum $0.3-0.6 \mathrm{MeV}$ by AVS-F data. The temporal profiles of the flare in various energy ranges are different each other. There are some $\gamma$-maxima (see Table 1 ) during this solar flare: at 20:42 UT (in energy ranges corresponding to continuum, ${ }^{56} \mathrm{Fe}$, ${ }^{24} \mathrm{Mg}+{ }^{20} \mathrm{Ne}+{ }^{28} \mathrm{Si}$ and ${ }^{12} \mathrm{C}$ ), 20:43 UT (in continuum and ${ }^{12} \mathrm{C}$ energy ranges), 20:48 UT 
Table 1. Spectral features of solar flare 29.10.2003 and temporal profile peaks position in the corresponding energy regions on AVS-F data

\begin{tabular}{|c|c|c|c|c|c|c|c|c|c|}
\hline $\begin{array}{c}\text { spectral } \\
\text { feature, } \\
\mathrm{MeV}\end{array}$ & $\begin{array}{l}\text { line } \\
\text { center, } \\
\text { MeV }\end{array}$ & interpretation & & & pea & ks posi & ition & & \\
\hline $0,3-0,6$ & - & continuum & $20: 42$ & $20: 43$ & - & $20: 46$ & - & $20: 51$ & - \\
\hline $0,81-0,94$ & 0,86 & $\mathrm{Fe}$ & $20: 42$ & - & $20: 45$ & $20: 46$ & $20: 48$ & $20: 51$ & - \\
\hline $1,51-1,74$ & 1,6 & $\mathrm{Mg}+\mathrm{Ne}+\mathrm{Si}$ & $20: 42$ & - & $20: 45$ & $20: 46$ & - & $20: 51$ & $20: 52$ \\
\hline $2,14-2,64$ & 2,2 & $\mathrm{n}$ & - & - & $20: 45$ & $20: 46$ & $20: 48$ & $20: 51$ & $20: 52$ \\
\hline $2,9-3,4$ & 3,1 & $\mathrm{Ne}+\mathrm{O}$ & - & - & - & - & - & - & $20: 52$ \\
\hline $4,0-5,0$ & 4,2 & C & $20: 42$ & $20: 43$ & $20: 45$ & $20: 46$ & $20: 48$ & $20: 51$ & - \\
\hline $5,3-6,9$ & 6,0 & $\mathrm{O}$ & - & — & $20: 45$ & $20: 46$ & $20: 48$ & $20: 51$ & - \\
\hline
\end{tabular}

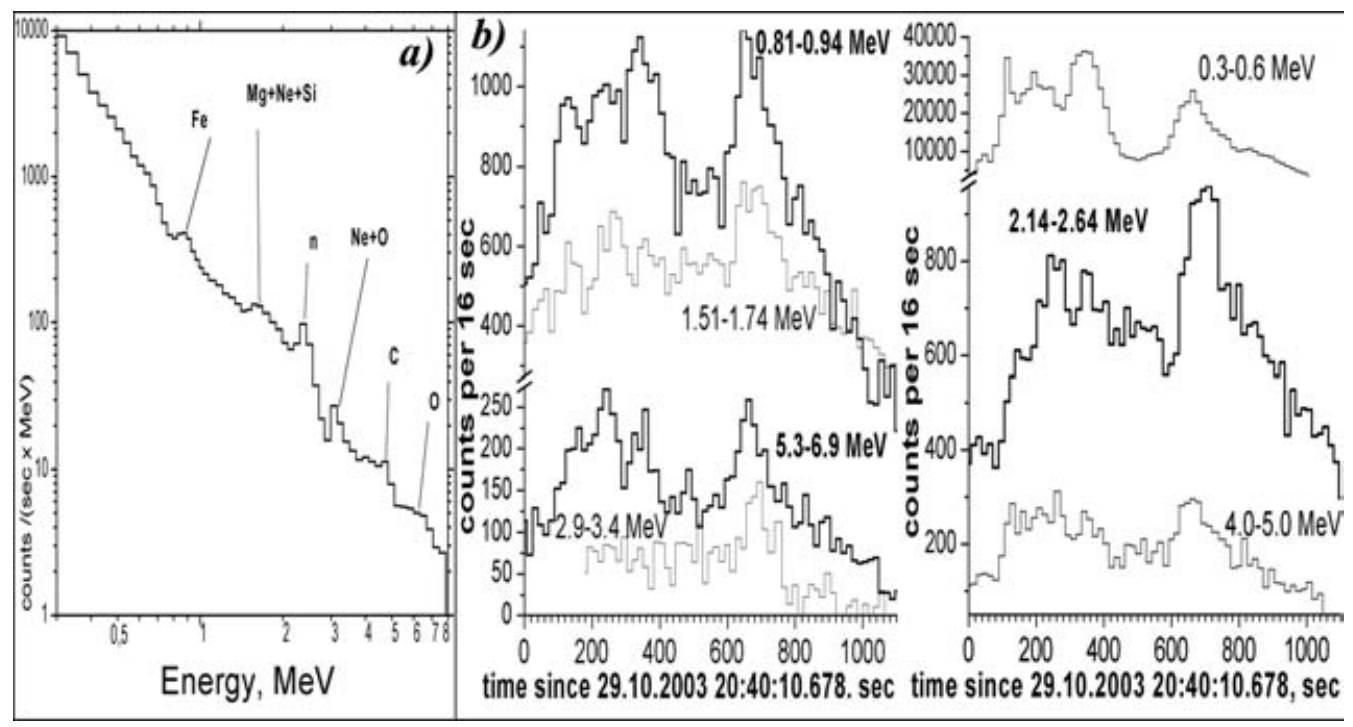

Figure 1. Spectrum in time interval 20:39:07-20:55:00 by AVS-F data (a) and temporal profiles in various energy ranges (b) for solar flare 29.10.2003

(in energy bands corresponding to ${ }^{56} \mathrm{Fe}$, n-capture, ${ }^{12} \mathrm{C}$ and ${ }^{16} \mathrm{O}$ ) and $20: 52 \mathrm{UT}$ (in ${ }^{24} \mathrm{Mg}+{ }^{20} \mathrm{Ne}+{ }^{28} \mathrm{Si}, \mathrm{n}$-capture and ${ }^{20} \mathrm{Ne}+{ }^{16} \mathrm{O}$ regions). The maximum at 20:45 UT was observed in all energy ranges except continuum and ${ }^{20} \mathrm{Ne}+{ }^{16} \mathrm{O}$ line. The maximum at 20:46 UT was observed in all energy ranges too except ${ }^{20} \mathrm{Ne}+{ }^{16} \mathrm{O}$ line. There were not any maxima in the any energy ranges corresponding to maximum at 20:49:00 UT in soft X-ray data of GOES-12. Indeed there was maximum at 20:48 UT corresponding to one detected in energy range $12-25 \mathrm{keV}$ by RHESSI (hesperia.gsfc.nasa.gov). Now we conduct the reconstruction and analysis of the other four flares observed by AVS-F during mentioned above time interval.

\section{References}

Arkhangelsky, A.I., Glyanenko, A. S., Kotov Yu. D., et al. 1999 IET $42 N^{\circ} \mathbf{5}, 596-603$.

http : //sec.noaa.gov/ftpdir/indices/2003_events/20030129events.txt Kuznetsov, S. N. et al. 1995 Izv.RAN, Physics series $59 N^{o} \mathbf{2}, 715-717$. http://hesperia.gsfc.nasa.gov/hessidata/dbase

Mandzhavidze, N. \& Ramaty, R. 1992 ApJ 389, 739-755. 